\title{
The contrasting impact of global and local object attributes on Kanizsa figure detection
}

\author{
Markus Conci and Hermann J. Müller \\ Ludwig-Maximilians-Universität München, Munich, Germany \\ AND \\ MARK A. ElliotT \\ Ludwig-Maximilians-Universität München, Munich, Germany \\ and National University of Ireland, Galway, Ireland
}

\begin{abstract}
Studies on the involvement of object completions in search for illusory figures have so far reported equivocal results. We have addressed this issue by investigating at which level object attributes in Kanizsa figures influence search. Employing a paradigm that investigated global and local attributes in the composition of distractors with relation to target composition, we report a selective involvement of multilevel processing upon detection. Four experiments demonstrate that global surface information, but not the surrounding global contour, determines the speed of Kanizsa figure detection. By contrast, local inducer information is encoded far less efficiently in search than processes computing the global object. Our conclusions are that surface filling-in acts as a major determinant of search, but depends on the relevance of the particular hierarchical level (local or global) coding the target.
\end{abstract}

When we perceive visual scenes, luminance discontinuities allow us to establish the boundaries between different objects. However, in many cases, the requisite information reaching the eye is incomplete. Adverse lighting conditions and overlapping objects may require the visual system to complete missing sensory information, to bring about the perception of a coherent object. Visual completion is demonstrated by the phenomenon of illusory figure perception (see Lesher, 1995, and Spillmann \& Dresp, 1995, for reviews). For example, in Figure 1A, a square is perceived to occlude the neighboring parts of the four circular inducer elements, even though there is no corresponding physical correlate (Kanizsa, 1955). Phenomenally, the illusory figure appears as a central bright region surrounded by sharp boundaries and depicting depth stratification.

Illusory figures, as with many other composite objects, result from the integration of the various parts into coherent wholes. As such, an integrated object may be represented at one of several levels in hierarchical organization. The Kanizsa square (Figure 1A) provides an example of a hierarchical stimulus configuration, comparable to the Navon letter (Navon, 1977; see the present Figure 1B). Both Kanizsa and Navon figures can be described at local and global levels of organization. For instance, at a local level, the Kanizsa figure is constructed from inducers just as the Hs in the Navon letter. In addition, at a global level, the Kanizsa figure consists of the illusory figure (the induced object) and the Navon figure of the global letter
"U" (see Figure 1). Thus, both objects are represented at multiple levels of representation, with global properties being dependent on the existence and arrangement of more elementary local parts.

Investigations of how a global object is derived from local elements show that several processes contribute to the final percept. Whereas the representation of local elements can be achieved in the main by luminance-selective units in early visual areas, global object properties necessitate more complex processes. For instance, the global letter in Figure 1B would result from grouping operations that link local elements. Similarly, the global square in the Kanizsa figure results from at least two independent processes, related to the extraction of contours and the specification of the illusory surface (Grossberg, 2000; Grossberg \& Mingolla, 1985, 1987). Thus, processes of object integration consist of multiple stages of processing (Lesher, 1995; Palmer, Brooks, \& Nelson, 2003) that can be related to distinct neural mechanisms: In neurophysiological studies, local luminance discontinuities are detected by cells in V1 (Hubel \& Wiesel, 1968). By contrast, the representation of a global object such as for the Kanizsa figure can be related to the computation of illusory contours in area V2 (Ffytche \& Zeki, 1996; Lee \& Nguyen, 2001; Peterhans \& von der Heydt, 1991; von der Heydt, Peterhans, \& Baumgartner, 1984), and corresponding surface filling-in mechanisms can be located further along the ventral stream in the lateral occipital complex and fusiform gyrus (Conci, Gramann,

M. Conci, conci@psy.uni-muenchen.de 
A
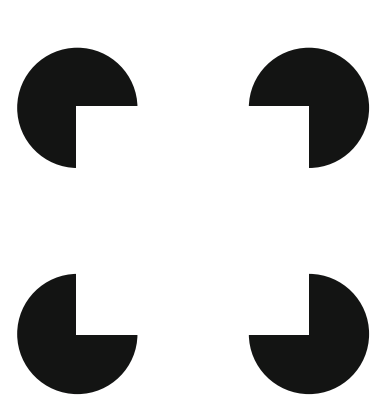

Figure 1. Examples of hierarchical stimuli: (A) Kanizsa figures (B) Navon letter.

Müller, \& Elliott, 2006; Hirsch et al., 1995; Murray et al., 2002 ; Stanley \& Rubin, 2003). Consequently, the representation of a hierarchical object can be attributed to several specialized processes. In the case of a Kanizsa figure, a representation of the local inducers may be distinguished from global (i.e., contour and surface) operations that induce the percept of an illusory figure (see Figure 2 for an illustration)

Studies that have investigated which hierarchical level determines behavioral performance have shown that only one level is relevant for perception at a given moment (Enns \& Kingstone, 1995), with precedence for the processing of global over local stimulus attributes (He \& Nakayama, 1992; Navon, 1977; Rauschenberger \& Yantis, 2001; Rensink \& Enns, 1995, 1998). However, global precedence has not been resolved unequivocally in the case of illusory figures in visual search. In the relevant studies, observers had to discern the presence of an illusory target figure as fast as possible from among a set of distractors composed of the same inducer elements, which were, however, rearranged so as not to form a global object (see Figure 4B for an example display). The results led some authors to propose that search is based upon completion of global illusory contours (Davis \& Driver, 1994; see also Gurnsey, Humphrey, \& Kapitan, 1992). By contrast, others have argued that there is no evidence to support the idea that search for Kanizsa figures is based on a global object representation (Grabowecky \& Treisman, 1989; Gurnsey, Poirier, \& Gascon, 1996). In the study of Gurnsey et al. (1996), for example, search efficiency clearly depended on factors other than the presence of illusory contours. Consequently, the role of global object representations in illusory figure search remains uncertain, since factors other than illusory contour and surface coding may influence the efficiency of search.

Different interpretations may, at least in part, arise from differing conceptualizations of how the various processing stages involved in object completion are integrated over time. On the one hand, extraction of local (inducer) and global (contour and surface) object properties may be considered as a strict feedforward process. In this view, perception of a Kanizsa figure would involve the integration of information in a series of processing steps, with higher levels of complete object representation reached only after termination of lower level stages. Figure 3A il- lustrates a corresponding model of processing. In a first step, local edge information is extracted. Next, illusory contours are computed, signaling precise borders to the subsequent process accomplishing surface filling-inthe final step in creating a complete object representation. In contrast, in a recurrent network, completion of an illusory figure would result from a series of feedforward and feedback loops with processing operating in parallel across different levels in the hierarchy (see Grossberg, 2000; Grossberg \& Mingolla, 1985; Roelfsma, Lamme, Spekreijse, \& Bosch, 2002). Figure 3B illustrates this model of processing. Here, processing is initiated by the specification of local edges, while subsequently global object properties are extracted by independent contourand surface-processing mechanisms. The outputs from the contour- and surface-processing mechanisms are then adjusted such that the relatively crude specification of the surface is fitted to the exact contour outlines, thus providing the final complete object representation.

In summary, hierarchical object computations may be conceptualized in different ways depending on how the processes involved are thought to engage in the extraction of specific object properties: Feedforward models envisage sequential object completion stages; in contrast, recurrent models of completion assume that different object attributes are extracted independently of each other and information from the different processes is integrated by recurrent matching of outputs only subsequently. Both models may eventually lead to differential predictions. For instance, on a strict feedforward account, a global object could be extracted only if global contours are specified beforehand. By contrast, on a recurrent network account, different object properties may be specified relatively independently of each other. Thus, surface information may be generated without an exact specification of the illusory contours that demarcate the object boundaries. In this view, selection processes would not necessarily have to rely on

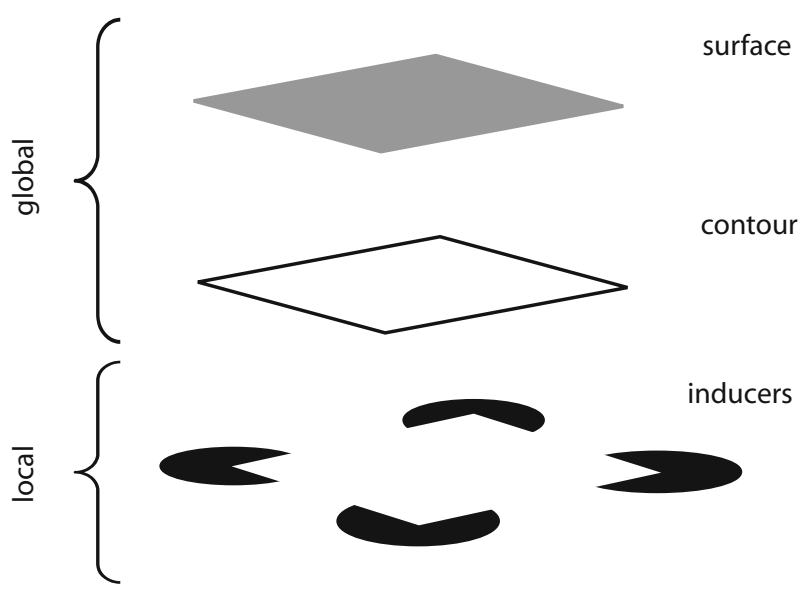

Figure 2. Illustration of local and global perceptual representations of an illusory Kanizsa figure. The local level consists of physically specified inducer elements. At the global level, an emergent square arises with contributions from two separate mechanisms that interpolate illusory contours and fill-in surface information (see Grossberg \& Mingolla, 1985). 
A

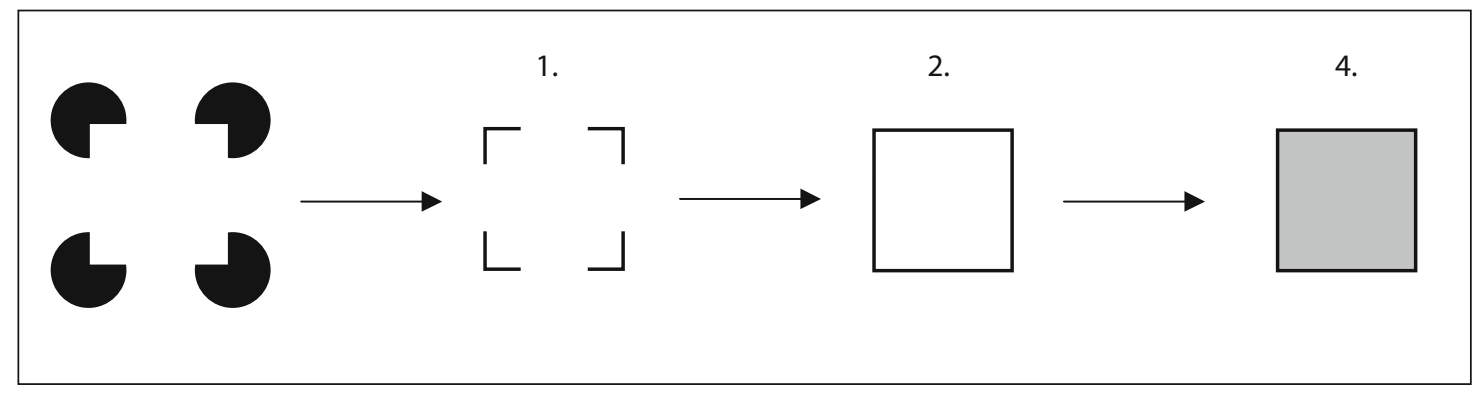

B

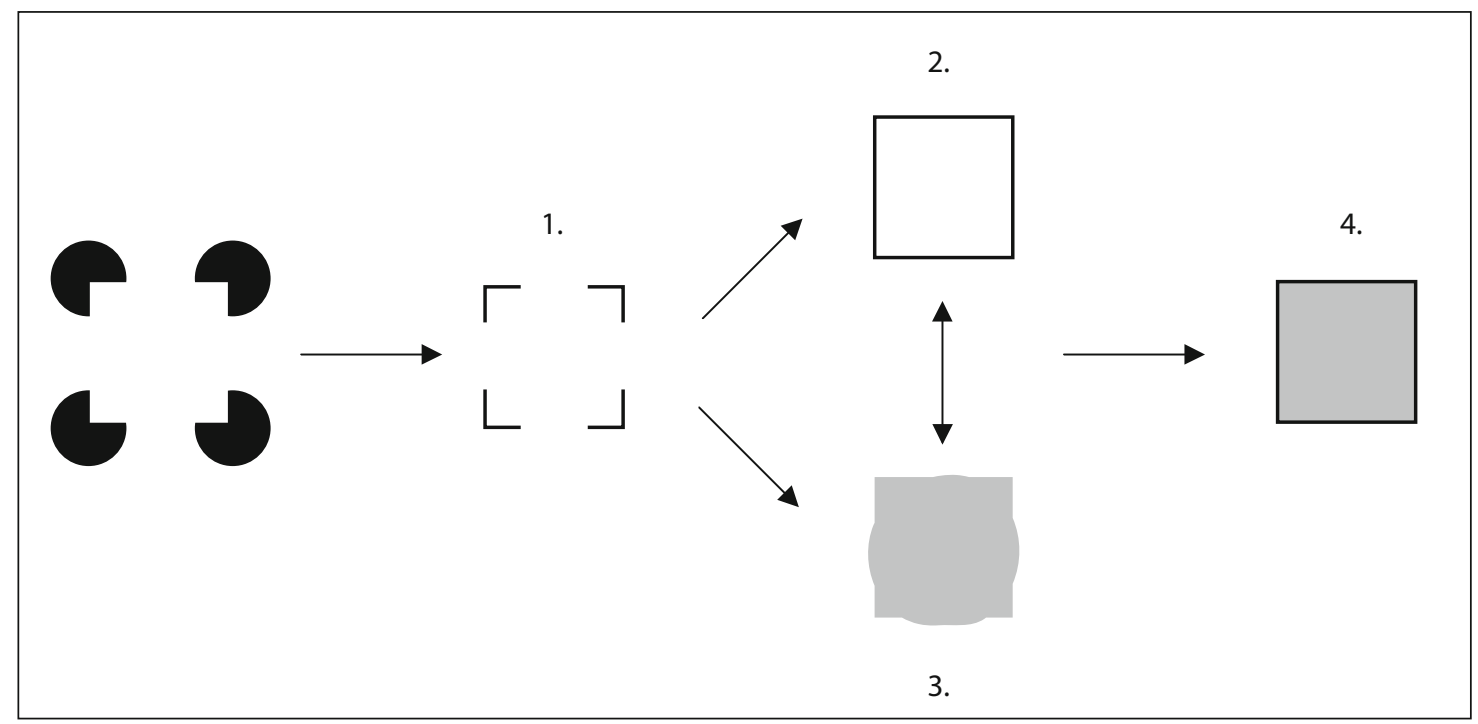

Figure 3. (A) Feedforward and (B) recurrent models of illusory-figure completion. Both models initially extract local edge information (1). Subsequently, (A) sequential or (B) parallel processes of global contour interpolation (2) and surface filling-in (3) match their outputs to form an integrated global object representation (4).

the final integrated output. Rather, in order to detect a given target configuration, a relatively crude representation of an object might provide the information required for a response. For example, the relatively crude segmentation of the central region within Kanizsa-type figures has been shown to be sufficient for efficient detection performance (Conci, Müller, \& Elliott, 2007; Stanley \& Rubin, 2003).

In order to decide between the alternative conceptualizations of feedforward and recurrent processing (see above) and to determine which aspect of an illusory figure can be regarded as critical for successful detection of a Kanizsa figure, we carried out a series of reaction time (RT) visual search experiments (see Conci et al., 2007, for a similar approach). In these experiments, observers had to report, as rapidly and accurately as possible, the presence or absence of a Kanizsa square target (as illustrated in Figure 1A) among different types of distractor configurations composed of similar local inducers. The distractor configurations were systematically varied in the degree to which global object properties could serve as a cue for search. This permitted exploration of whether systematic variations of global distractor attributes would lead to in- terference in search for a global target configuration (cf. Duncan \& Humphreys, 1989).

Experiment 1 compared the efficiency of detecting a Kanizsa target square among distractors consisting of similar local inducers, but arranged to form dissimilar global objects (configurations). Global distractor objects rendered either only contour (border-type distractors) or contour plus surface information (form-type distractors). The terms border and contour are meant to refer to a single process: global contour interpolation. Figure 4A presents the various configurations for the two (form and border) distractor types (each configuration is depicted as an arrangement of local inducers; in addition, the corresponding global objects are illustrated according to the hierarchical levels sketched in Figure 2); Figure 4B presents an example search display. The results of the experiments revealed that only distractors providing global surface, but not those providing global contour, information interfered with target detection by slowing search rates (i.e., the slope of the function relating search RT to display size). This strongly suggests that mechanisms filling in surface information play a role in search performance. 
Based on this finding, Experiment 2 was designed to further examine whether the computation of global surfaces and of contours are dependent on one another, or whether they are dissociable. This was done by creating distractors with systematic variations of contours independently of their surface characteristics (see Figure 6 for example distractor types). Again, the results showed that only the specification of surface information in distractors interfered with search. That is, the computation of surfaces may be to some extent independent of that of boundary contours.

Experiment 3 introduced a target configuration defined solely by the local arrangement of inducers, without a corresponding global object representation. As in the previous experiments, the number of distractor inducers that matched the orientation of the target inducers was systematically varied (see Figure 8A for example target and distractor configurations). Search for a specific local arrangement of inducers permitted investigation of how variations of local object attributes in distractors influence performance, similar to the logic adopted for global object variations in Experiments 1 and 2. The results revealed search performance to be dependent on the local arrangement of distractor inducers, specifically: Search efficiency decreased as increasing numbers of local inducers in the distractors matched the orientation of those in the target. While this pattern is generally similar to global search performance in Experiments 1 and 2, search efficiency was markedly reduced overall compared to the previous experiments. This suggests that local search is more susceptible to the presence of interfering information than is global search.

Finally, Experiment 4 was carried out to investigate whether a gradual increase of interfering global information in a given display would influence search performance. Display size was fixed to eight elements (including the target, if present). The distractors in a given display were composed of noninterfering placeholder configura- tions (with all inducers oriented such that no global target object was induced) and interfering configurations of the same type (with a fixed number of configurations that induced global surface and/or contour information matching the global target object). In addition, the number of interfering distractors that replaced placeholder distractors was systematically varied (interference set). Thus, the amount of interfering global surface and contour information in distractors was systematically varied such that the influence of global object attributes could be investigated in a similar fashion to Experiment 1 . The results replicated Experiment 1 in revealing the search rates (the slope of the function relating RT to interference set size) to be determined by global surface characteristics. Furthermore, contour information was found to influence the base RTs (i.e., the $y$-intercept of the function relating RT to interference set size). This indicates that, while global contours were encoded, only global surface variations affected search efficiency.

In sum, all four experiments revealed consistent evidence to suggest that filling-in mechanisms are predominantly guiding search for Kanizsa figures, with search efficiency depending on surface specifications and the number of candidate global objects in the visual display.

\section{EXPERIMENT 1}

Experiment 1 examined the extent to which variations in global contour and surface properties of distractors modify the efficiency of target Kanizsa figure detection. Observers performed a visual search task in which a Kanizsa figure had to be detected among distractors that provided either only contour information (see Figure 4 for example target [T] and border-type distractor [D] configurations) or both contour and surface information (see Figure 4 for example target $[\mathrm{T}]$ and form-type distractor [D] configu-
A

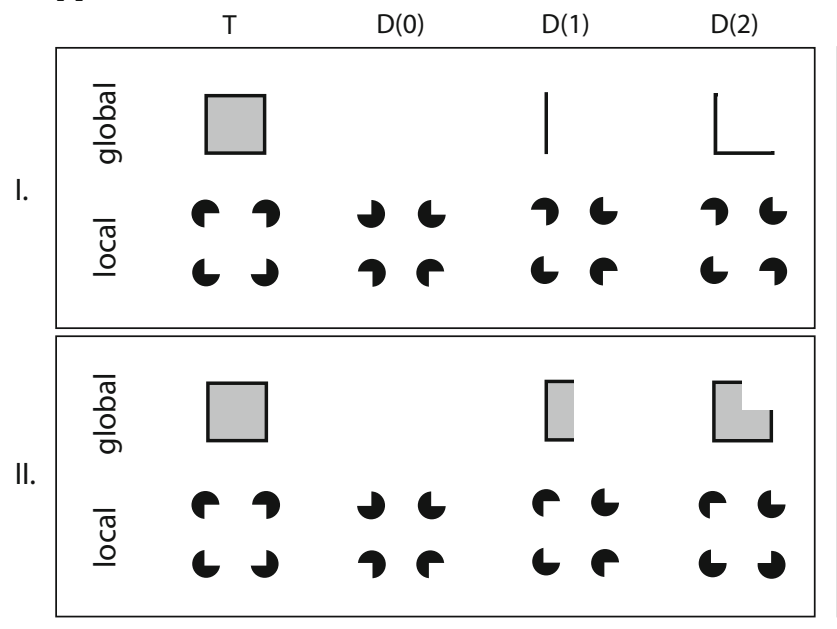

Figure 4. (A) Examples of the target Kanizsa square (T) and distractor stimuli (D) presented in Experiments 1 and 4. For each configuration, local (inducer) and global (contour and surface) representations are illustrated. Distractors could be constructed from zero, one or two illusory contours $(D(0)-D(2))$ that could either induce a global border (I) or a global form (II). (B) Example of a target present display with eight candidate groupings. 
rations). With both types of distractor configuration, the number of illusory contours increased from 0 (common baseline) through 1 to 2 (see Figure 4 for example configurations). The two types of distractor configuration differed in that neighboring illusory contour inducers were either both oriented toward the inside of the configuration (form-type distractors) or one oriented inward and the other outward (border-type distractors). Distractor configurations were chosen on the basis of neurophysiological work that has revealed the two types of configuration to elicit equivalent levels of activation in early visual areas $\mathrm{V} 1$ and V2, though the activation was reduced compared to that generated by a Kanizsa square (Lee \& Nguyen, 2001). This pattern of activations suggests that form- and border-type distractors elicit roughly similar responses in areas sensitive to the presence or absence of an illusory contour. From a computational viewpoint, the strength of the induced contours should be similar for the two types of distractor, as direction of contrast is not crucial for contour formation (Grossberg \& Mingolla, 1985). In addition, both subjective stimuli that emerge due to changes in contrast polarity such as offset gratings and stimuli that rely on similar directions of contrast such as Kanizsa figures do elicit illusory contours (see Grossberg, 2000), and both types of configuration have been shown to affect search performance at parallel stages of processing (Davis \& Driver, 1994; Gurnsey et al., 1992). On these grounds, the form- and border-type configurations per se may be considered to be reasonably comparable. Thus, the systematic manipulation of global object attributes in distractors was designed to establish whether a gradual increase of global figural properties (contours and surfaces) would influence - that is, interfere with - detection of a target Kanizsa figure.

\footnotetext{
Method

Participants. Eight paid observers ( 2 of whom were male, mean age 27.1 years) with normal or corrected-to-normal visual acuity participated in the experiment for payment of $€ 8$ per hour.

Stimuli. Stimuli were generated using an IBM-PC compatible computer and presented in light gray $\left(1.83 \mathrm{~cd} / \mathrm{m}^{2}\right)$ against a black $\left(0.02 \mathrm{~cd} / \mathrm{m}^{2}\right)$ background at 8 possible locations on a 17 -in. monitor screen. The stimulus configurations were placed on a virtual circle around the screen center, with radius $8.75^{\circ}$ of visual angle (at a viewing distance of $55 \mathrm{~cm}$ ). An example display with 8 stimulus configurations is shown in Figure 4B. Each configuration or candidate grouping was composed of 4 inducing elements with a diameter of $1^{\circ}$ arranged in a square-like form that subtended a viewing angle of $2.9^{\circ} \times 2.9^{\circ}$. As depicted in Figure 4A, the target $(\mathrm{T})$ was defined as a Kanizsa square. In contrast, distractor configurations (D) were produced by rotating inducer elements such that each grouping contained only zero, one, or two aligned illusory contours. In this way, 2 types of distractor configurations were generated: partial global form groupings (containing contour and surface information) and partial global border groupings (depicting only contour information); for examples, see Figure 4. Trial displays could contain 1, 2, 4 , or 8 candidate groupings (the display size), with each grouping presented in a random orthogonal orientation. In $50 \%$ of the trials, a target configuration was present in the display. For displays with fewer than 8 candidate groupings, the stimulus positions were chosen pseudorandomly from among the 8 possible locations with the following constraints: For display sizes of 2, candidate groupings were presented at diametrically opposite positions only; and for dis-
}

play sizes of 4 , groupings were presented at every second position of the 8 possible locations.

Procedure. Each trial started with the presentation of a central fixation cross for $500 \mathrm{msec}$. The fixation cross was then immediately replaced by the search display, to which observers responded with a speeded target-absent/present response via pressing the left/ right mouse button. Displays remained on screen until a response was recorded. In case of an erroneous response or a time-out (i.e., after a period of 2,500 msec without reaction), feedback was given by a computer generated tone and an alerting message was presented for $500 \mathrm{msec}$ at the center of the screen. Each trial was separated from the next by an interval of $500 \mathrm{msec}$.

The experiment was conducted over two sessions, each presenting either global-border or global-form distractors. Each session consisted of 12 blocks of 80 trials, with the number of illusory contours $[D(0), D(1), D(2)]$ constant throughout a block. Blocks were administered in pseudorandom order on an observer-by-observer basis. In summary, the independent variables were: target ( $\mathrm{T}$ : present, absent), display size (DS: 1, 2, 4, 8 configurations), distractor type (DT: global-border, global-form), and illusory contours in distractor configurations (IC: $0,1,2$ contours), with 40 trials per condition.

\section{Results and Discussion}

RT analysis. RTs on trials on which a response error was made $(4.1 \%)$ were removed from the data set prior to RT analysis. Figure 5 presents the mean correct RTs and the percentage of errors as a function of display size, separately for the global-border and global-form distractors (see figure columns a and $b$, respectively) and the various illusory contour (i.e., IC) conditions.

The whole data set was initially examined by a repeated measures ANOVA, with the factors distractor type, illusory contours, target, and display size. This ANOVA revealed all main effects and interactions, including the four-way interaction $[F(6,280)=6.14, p<.001]$ to be significant. Next, to decompose the four-way interaction, the data sets for global-border and global-form distractor types were analyzed separately by two ANOVAs with the factors illusory contours, target, and display size.

For global-border distractors, the ANOVA revealed all main effects $[\mathrm{T}, F(1,7)=20.08, p<.01$; DS, $F(3,21)=$ $17.35, p<.001$; IC, $F(2,14)=17.11, p<.001]$ and interactions with display size to be significant $[\mathrm{T} \times \mathrm{DS}$, $F(3,119)=13.58, p<.001 ; \mathrm{DS} \times \mathrm{IC}, F(6,119)=6.21$, $p<.001]$. Target-present RTs were overall faster than target-absent RTs; and RTs increased as a function of display size, with a somewhat more marked increase for target-absent relative to target-present RTs (DS effects of 28.0 vs. $15.3 \mathrm{msec} /$ item). In addition, RT increases as a function of display size were more pronounced for $\mathrm{D}(1)$ and $\mathrm{D}(2)$ distractors than for $\mathrm{D}(0)$ distractors (DS effects of 25.7 vs. $13.5 \mathrm{msec} /$ item). However, despite the significant display size effects, the search rates for targetpresent trials ( $17.5 \mathrm{vs}$. $11.0 \mathrm{msec} /$ item $)$ indicated that the search could be performed with moderate efficiency.

By contrast, for global-form distractors, the ANOVA revealed all effects to be significant $[\mathrm{T}, F(1,7)=87.35$, $p<.001$; DS, $F(3,21)=216.90, p<.001$; IC, $F(2,14)=$ $141.93, p<.001 ; \mathrm{T} \times \mathrm{DS}, F(3,119)=82.52, p<.001$; $\mathrm{T} \times \mathrm{IC}, F(2,119)=52.40, p<.001 ; \mathrm{DS} \times \mathrm{IC}, F(6,119)=$ $120.04, p<.001 ; \mathrm{T} \times \mathrm{DS} \times \mathrm{IC}, F(6,119)=14.91, p<$ $.001]$. As can be seen from Figure $5 \mathrm{~B}, \mathrm{RT}$ s increased mark- 
A
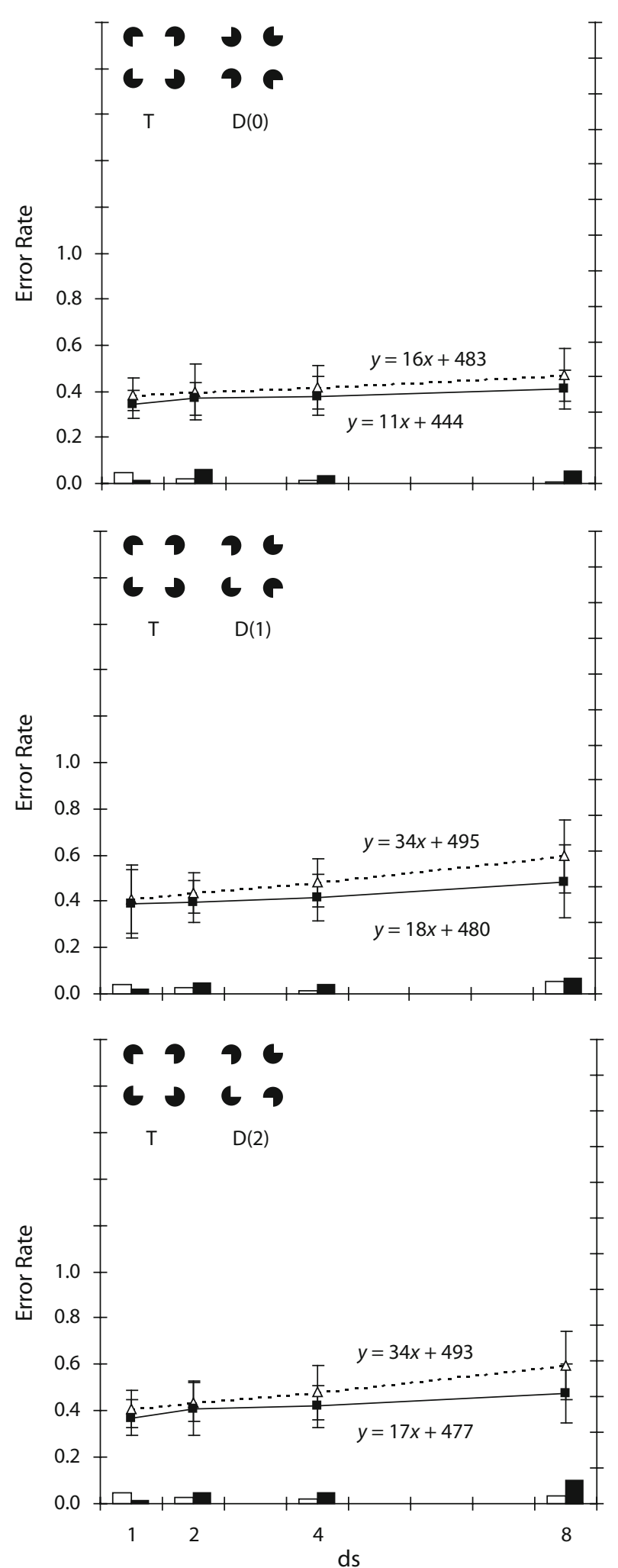

B
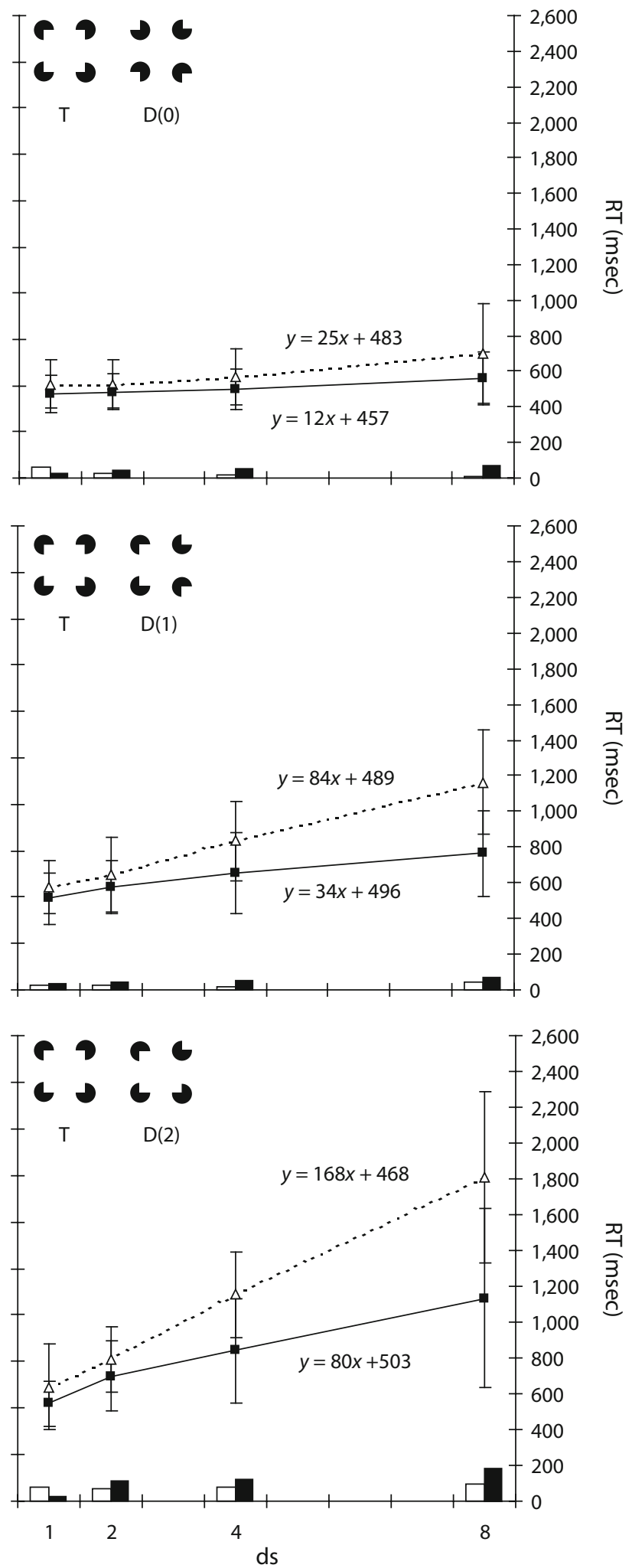

Figure 5. Mean RTs (with associated SDs) and error rates in Experiment 1 as a function of display size (ds), separately for (A, left column) global-border and (B, right column) global-form distractors with zero, one, and two contours (top, middle, and bottom graphs, respectively). Each graph shows the prototype target (T) and an example of a distractor (D) and plots RTs and error rates separately for target-absent (dotted line, white bars) and target-present trials (solid line, black bars). In addition, the function for the best fitting straight line is given for each search RT function, with the search rate and base RT estimates. 
edly with increasing display size as the number of illusory contours in distractor configurations increased, with search rates (target-absent [target-present]) decreasing from 25 [12] through 84 [34] to 168 [80] msec/item with zero, one, and two contours, respectively. The significant four-way ANOVA in the overall analysis is thus accounted for by the fact that this pattern of increasing interference, associated with increasing number of illusory contours in distractor configurations, was manifest only for globalform, but not for global-border, distractor types.

Error analysis. RTs on trials on which a response error was made tended to be overall slower than correct RTs, which argues against RT performance being contaminated by speed-accuracy tradeoffs (this was the case in all subsequent experiments as well).

Overall, errors were relatively rare $(4.9 \%$ misses; $3.2 \%$ false alarms). The arcsine-transformed error rates were examined by ANOVAs of the same design as those applied to the RT data. The overall ANOVA failed to reveal the four-way interaction to be significant. For global-border stimulus configurations, the ANOVA revealed significant effects for target and display size [T, $F(1,7)=6.57, p<$ .05 ; DS, $F(3,21)=3.23, p<.05$; T $\times$ DS, $F(3,119)=$ $11.18, p<.001]$. Error rates, in particular miss rates increased with display size, and target misses (target-absent trials) were more frequent than false alarms (target-present trials), despite the fact, mentioned above, that no speedaccuracy tradeoffs were evident.

For global-form distractors, the ANOVA revealed a significant increase in error rates with display size, in particular for target miss errors [DS, $F(3,21)=3.49, p<.05$; T $\times$ DS, $F(3,119)=6.12, p<.001]$. In addition, error rates were affected by the number of illusory contours [IC, $F(2,14)=$ $29.90, p<.001]$. Thus, in the global-form condition, increasing the number of illusory contours in distractors produced increasing interference with search performance not only in terms of response speed, but also response accuracy. This pattern is in contrast with performance in the globalborder condition, in which performance was unaffected by the number of illusory contours in distractors.

Discussion. Experiment 1 revealed the efficiency of target detection to be relatively unaffected by the presence of global-border distractors in the display: Search was close to optimal efficiency (i.e., parallel) in all conditions (across conditions, the search rates averaged 15.3 [28.0] $\mathrm{msec} / \mathrm{item}$ ), and the number of illusory contours had only a small effect upon search (each additional contour slowed search by 3.0 [9.0] msec/item). This slowing of search performance was more pronounced for the difference between baseline $\mathrm{D}(0)$ and $\mathrm{D}(1)$ distractors. In contrast, performance for $\mathrm{D}(1)$ distractors was near-equal to that for $\mathrm{D}(2)$ distractors. One possible explanation for this difference between $\mathrm{D}(0)$ groupings on the one hand and $\mathrm{D}(1)$ and $\mathrm{D}(2)$ groupings on the other may be that it was not the contours which were critical for differences between conditions, but simply the reduction of symmetry between zero- and one- or two-contour distractors.

In contrast to the relatively efficient performance for global-border distractors, global-form distractors significantly and systematically affected target search rates, with each additional contour in the distractors slowing search per item by 34.0 [71.5] msec (as compared with 3.0 [9.0] msec with global-border distractors). As a result, with one and two contours, the search rates (34 [84] and 80 [168] msec/item, respectively) were outside the range taken to be indicative of efficient search.

In summary, Experiment 1 revealed that search differs markedly between global-border and global-form distractors: Only global-form distractors interfere with target detection, with the degree of interference depending on the number of illusory contours. This pattern suggests that, while both distractor types share identical boundaries, interference is observed only when distractors exhibit surface information.

\section{EXPERIMENT 2}

Experiment 1 revealed search efficiency to be influenced by surface information, but not by contour information. However, while variations in the distractor contours did not affect search efficiency, when the contours varied together with corresponding surface portions, there was a clear effect on performance. According to computational models, surface filling-in and contour completions are achieved by separate subsystems (Grossberg \& Mingolla, 1985). Consequently, Experiment 2 was conducted to more closely investigate whether the co-occurrence of contours and surfaces in the global-form condition is crucial for the observed influence on performance. Of course, disentangling these two factors is difficult since changes in one aspect of a configuration may alter other aspects as well. Nevertheless, Experiment 2 sought to dissociate the two factors by varying the surrounding contours while maintaining a specific definition of the surface (see Fig-

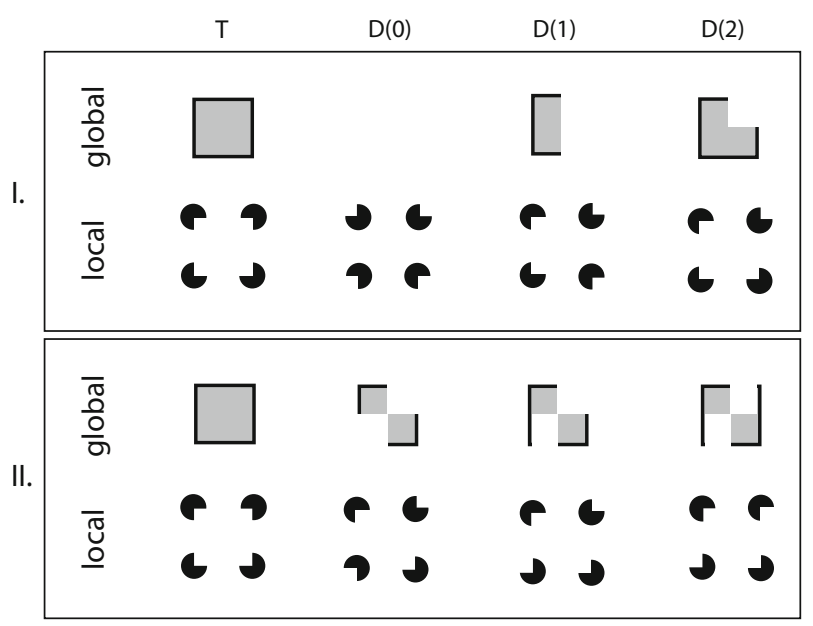

Figure 6. Examples of the target Kanizsa square (T) and distractor configurations presented in Experiment 2. For each configuration, local (inducer) and global (contour and surface) representations are illustrated. Distractors could contain zero, one or two illusory contours $(\mathrm{D}(0)-\mathrm{D}(2))$. The upper panel depicts stimuli from the global-form condition (I) and the lower panel illustrates the corresponding global-surface condition (II) that varied global contours while controlling for global surface characteristics in distractors. 
ure 6). This global-surface condition was compared to the global-form condition (as in Experiment 1, see Figure 6), presenting global contour and surface information in relation to each other.

\section{Method}

This experiment was identical to Experiment 1, except that the global-border condition was replaced by the global-surface condition. Stimulus configurations for global-surface distractors were designed such that a constant global surface resulted from two inward-facing inducer elements placed on a diagonal while contour specifications were varied from zero through one to two along the vertical continuations of each configuration (see Figure 6). Eight paid observers ( 3 of whom were male, mean age 26.1 years) with normal or corrected-normal vision participated in the experiment. All other methodological details were the same as in Experiment 1.

\section{Results and Discussion}

RT analysis. As with Experiment 1, trials on which a response error was made $(3.1 \%)$ were removed from the data set prior to RT analysis. Figure 7 presents the mean correct RTs and the error percentages as a function of display size, separately for the global-form and global-surface distractor (see figure columns $a$ and $b$, respectively) and for the various illusory contour conditions. As can bee seen, the global-form condition (see Figure 7A) closely replicated the results from Experiment 1: Search efficiency decreased substantially with increasing surface (and contour) specification. By contrast, in the global-surface condition, no comparable variations were obtained (see Figure 7B).

The whole data set was initially examined by repeated measures ANOVA, with the factors distractor type, illusory contours, target, and display size. This ANOVA revealed all main effects and interactions, including the four-way interaction $[F(6,280)=7.24, p<.001]$, to be significant. To examine the four-way interaction, the data sets for global-surface and global-form distractor types were analyzed separately by two ANOVAs with the factors illusory contours, target, and display size. For globalform distractors, the ANOVA revealed all effects to be significant $[\mathrm{T}, F(1,7)=100.07, p<.001 ; \mathrm{DS}, F(3,21)=$ $70.83, p<.001$; IC, $F(2,14)=110.71, p<.001 ; \mathrm{T} \times$ DS, $F(3,119)=123.21, p<.001 ; \mathrm{T} \times \mathrm{IC}, F(2,119)=$ 42.74, $p<.001 ; \mathrm{DS} \times \mathrm{IC}, F(6,119)=91.48, p<.001$; $\mathrm{T} \times \mathrm{DS} \times \mathrm{IC}, F(6,119)=13.81, p<.001]$. As with Experiment 1 , an increase in the number of illusory contours produced an increase in the search time per item, which was significantly less pronounced for target-present than for target-absent trials. Each additional contour slowed the search time per item by 32.5 and $73.5 \mathrm{msec}$, respectively.

For global-surface distractors, by contrast, the ANOVA revealed the main effects for target and display size and their interaction to be significant $[\mathrm{T}, F(1,7)=56.72, p<$ $.001 ; \mathrm{DS}, F(3,21)=27.35, p<.001 ; \mathrm{T} \times \mathrm{DS}, F(3,119)=$ 90.78, $p<.001]$. Target-present RTs were faster than target-absent RTs; and RTs increased as a function of display size, with a steeper increase for target-absent relative to target-present RTs (DS effects of 88.3 vs. $39.0 \mathrm{msec} /$ item). In addition, target interacted with illusory contours $[\mathrm{T} \times \mathrm{IC}, F(2,119)=4.43, p<.05]$, due to a slight increase of target-absent search RTs with an increasing number of contours, as opposed to a decrease for targetpresent search RTs. In the global-surface condition, the search rates were independent of the number of illusory contours, with search efficiency being comparable to performance for $\mathrm{D}(1)$ distractors in the global-form condition [DS effects of $61.1 \mathrm{vs} .88 .5 \mathrm{msec} /$ item; $t(14)=1.43, p=$ .17].

Error analysis. Errors were relatively rare $(3.8 \%$ misses; $2.4 \%$ false alarms). The arcsine-transformed error data were analyzed by means of identical ANOVAs to those applied to the RT data. For global-form conditions, the ANOVA revealed significant main effects for display size and illusory contours [DS, $F(3,21)=5.95$, $p<.01 ; \mathrm{IC}, F(2,14)=23.19, p<.001]$, and significant interactions of illusory contours with target and with display size $[\mathrm{T} \times \mathrm{IC}, F(2,119)=4.29, p<.05 ; \mathrm{DS} \times \mathrm{IC}$, $F(6,119)=3.28, p<.01]$. For global-surface conditions, the ANOVA revealed similar main effects to be significant $[\mathrm{DS}, F(3,21)=3.64, p<.05 ; \mathrm{IC}, F(2,14)=8.94, p<.01]$ as well as the target $\times$ display size interaction $[\mathrm{T} \times \mathrm{DS}$, $F(3,119)=6.39, p<.001]$. In summary, error rates - in particular, miss rates - increased with both display size and the number of illusory contours (with roughly similar patterns for global-form and global-surface conditions). Speed-accuracy tradeoffs were not evident.

Discussion. To summarize, Experiment 2 replicated the results of Experiment 1 in showing that surface characteristics in the global-form condition influence search efficiency. When the surface (and contour) specification of distractors was increased, search became increasingly less efficient. Each additional contour, together with the addition of corresponding surface portions, slowed search by 32.5 [73.5] msec/item, which compares well with the 34.0 [71.5] msec/item found in Experiment 1. By contrast, in the global-surface condition, no comparable effect could be observed. The addition of contour information independent of surface specifications did not lead to a slowing of search ( -2.0 [3.0] msec/item). Instead, search performance for the global-surface condition remained equivalent across variations in the number of contours (see Figure 7B), with search rates being statistically indistinguishable from the global-form $\mathrm{D}(1)$ condition in which distractors supported a similar proportion of the surface (see Figure 7A). This outcome provides support for independent processes relating to surface filling-in and the specification of boundary contours, consistent with models in which contour and surface information are assumed to be computed in parallel by separate, interactive subsystems (see, e.g., Grossberg \& Mingolla, 1985). Given that global contour information does not influence search, the estimation of surface portions appears to be sufficient for deciding whether a search array contains Kanizsa target figure.

\section{EXPERIMENT 3}

Experiments 1 and 2 show that the efficiency of detecting an illusory figure does not depend upon contours, but rather on the specification of surface information. Surface- 
A
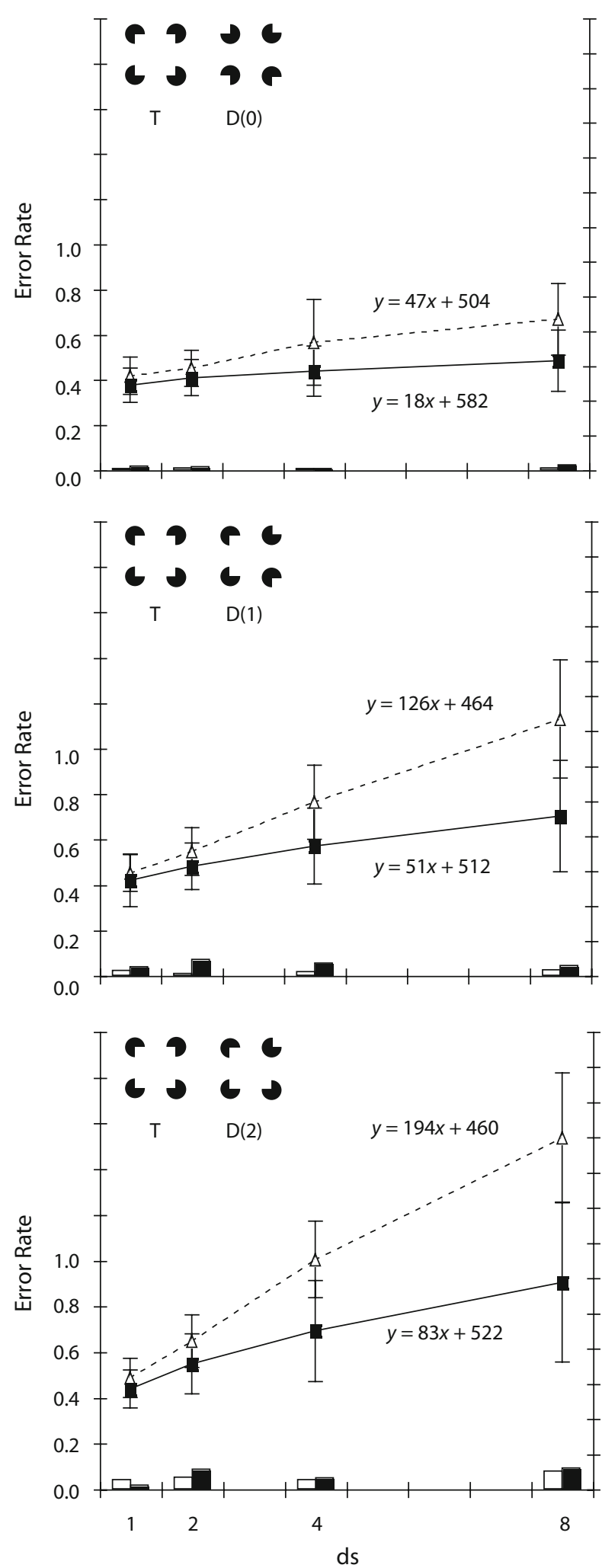

B
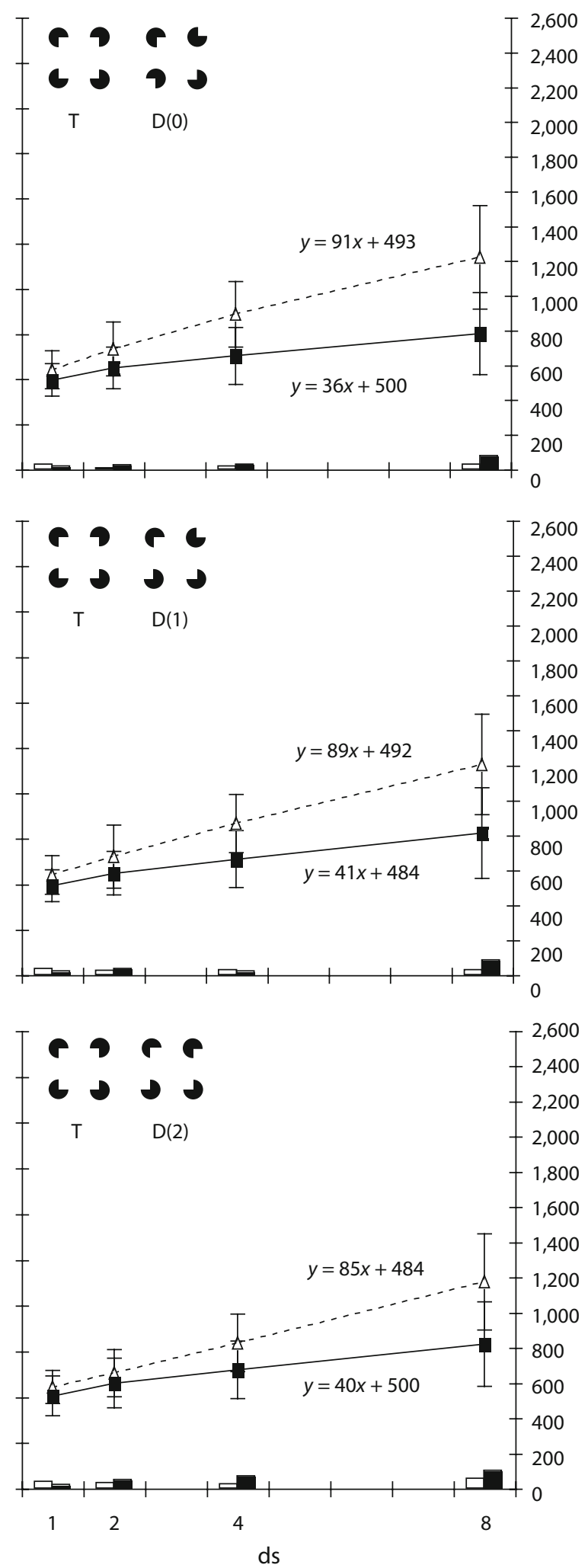

Figure 7. Mean RTs (with associated SDs) and error rates in Experiment 2 as a function of display size (ds), separately for (A, left column) global-form and (B, right column) global-surface distractors with zero, one, and two contours (top, middle, and bottom graphs, respectively). Each graph shows the prototype target (T) and an example of a distractor (D) and plots RTs and error rates separately for target-absent (dotted line, white bars) and target-present trials (solid line, black bars). In addition, the function for the best fitting straight line is given for each search RT function, with the search rate and base RT estimates. 
A

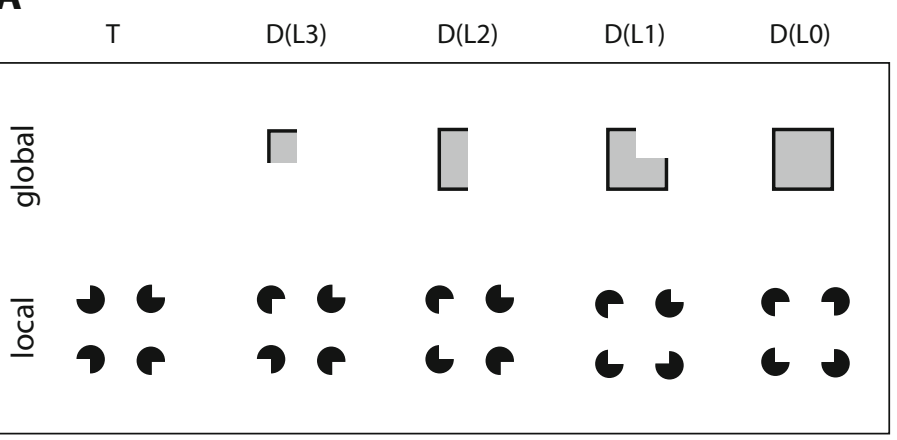

B

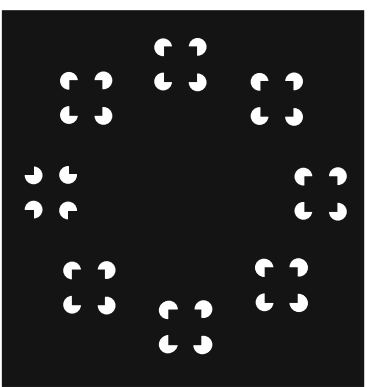

Figure 8. (A) Examples of the nonsquare target configuration (T) and distractors (D) presented in Experiment 3. Distractors could contain three to zero inducers that matched the orientation of local $(L)$ inducers with the target $[D(L 3)-D(L 0)]$. (B) An example target present display with eight candidate groupings.

specific interference operates at global object levels and leads to inefficient performance with prolonged response latencies and increased error rates. To compare global processing with the processing of local stimulus attributes, Experiment 3 examined the influence of local object specification upon search. For this purpose, a novel target configuration was used which is represented only at a local object level (a local nonsquare). This nonsquare had to be detected among distractors that varied local inducer orientation with relation to the target configuration (see Figure 8A for example target [T] and local-inducer distractor [D] configurations). This variation permitted investigation of the impact of local orientation matches on target detection.

\section{Method}

The experiment was in principle identical to the previous search experiments, except that the target was now defined as a nonsquare with inducing elements rotated outward by $180^{\circ}$ (see Figure $8 \mathrm{~A}, \mathrm{~T}$ ), resulting in a local-level stimulus representation. Distractors were varied comparably to the previous experiments, however, with local inducer orientations being matched in relation to the new target configuration. Thus, a distractor configuration could be designed from zero (baseline), one, two, or three inducing elements that would correspond to the local orientation of the target configuration [see Figure 8A; D(L0), D(L1), D(L2) and, D(L3), respectively; "L" refers to "Local"]. An example display with eight stimulus configurations is shown in Figure 8B. Eight paid observers (3 of whom were male, mean age 26.2 years) with normal or corrected-normal visual acuity participated in the experiment.

The experiment consisted of two sessions with eight blocks of 80 trials each. In summary, the independent variables were: target (T: present, absent), display size (DS: 1, 2, 4, 8 configurations), and local inducers in distractor configurations (LI: 0, 1,2,3 element-orientation matches), with 40 trials per experimental condition. All other details were identical to the procedure described for Experiment 1.

\section{Results and Discussion}

RT analysis. RTs on trials on which a response error was made $(4.6 \%)$ were removed from the data set prior to RT analysis. Figure 9 presents the mean correct RTs and the percentage of errors as a function of display size, separately for the various local orientation matches in distractors. As can be seen, an increase of local orientation matches in distractors resulted in a pronounced reduction of search efficiency.
The RT data were examined by a repeated measures ANOVA with the factors target, display size, and local inducers. The ANOVA revealed all effects to be significant $[\mathrm{T}, F(1,7)=104.79, p<.001 ; \mathrm{DS}, F(3,21)=108.35, p<$ $.001 ; \mathrm{LI}, F(3,21)=125.21, p<.001 ; \mathrm{T} \times \mathrm{DS}, F(3,168)=$ $132.71, p<.001 ; \mathrm{T} \times \mathrm{LI}, F(3,168)=29.53, p<.001$; $\mathrm{DS} \times \mathrm{LI}, F(9,168)=105.86, p<.001 ; \mathrm{T} \times \mathrm{DS} \times \mathrm{LI}$, $F(9,168)=6.37, p<.001]$. An increase in the number of inducer elements matching in terms of the local orientation between target and distractors led to a steep decline in search efficiency. This decrease was more marked for target-absent than for target-present trials. Each additional local-inducer match slowed search by 53.3 [84.3] msec/ item.

In a subsequent step, differences between local and global levels in search were evaluated by comparing Experiment 1 with Experiment 3. The RT data from the global-form condition with baseline- $\mathrm{D}(0)$ and $\mathrm{D}(1)$ distractors in Experiments 1 were compared to the same conditions in Experiment 3 (that is, conditions with $\mathrm{D}(\mathrm{L} 0)$ and $\mathrm{D}$ (L2) distractors). Responses were collapsed in a mixeddesign ANOVA with the within-subjects factors target, display size, and distractor type and the between-subjects factor Experiment. The ANOVA revealed all main effects and interactions, including the four-way interaction $[F(3,42)=3.38, p<.03]$, to be significant. This indicates that search was overall less efficient in Experiment 3 than in Experiment 1 (mean RTs were 942 vs. $629 \mathrm{msec}$, respectively), with interference from distractor type variations leading to a larger increase in RTs with display size and target in Experiment 3, as compared with Experiment 1.

Error analysis. Response errors were relatively rare overall (7.2\% misses; $2.2 \%$ false alarms). The arcsinetransformed error data were analyzed by means of an identical ANOVA to that of the RT data. This analysis again revealed all effects to be significant $[\mathrm{T}, F(1,7)=9.96, p<$ .05 ; DS, $F(3,21)=14.42, p<.001$; LI, $F(3,21)=5.44$, $p<.01 ; \mathrm{T} \times \mathrm{DS}, F(3,168)=7.58, p<.001 ; \mathrm{T} \times \mathrm{LI}$, $F(3,168)=4.24, p<.01 ; \mathrm{DS} \times \mathrm{LI}, F(9,168)=2.18$, $p<.05 ; \mathrm{T} \times \mathrm{DS} \times \mathrm{LI}, F(9,168)=2.31, p<.05]$. Error rates - in particular, miss rates - increased as a function of display size and with an increase in the number of matching 

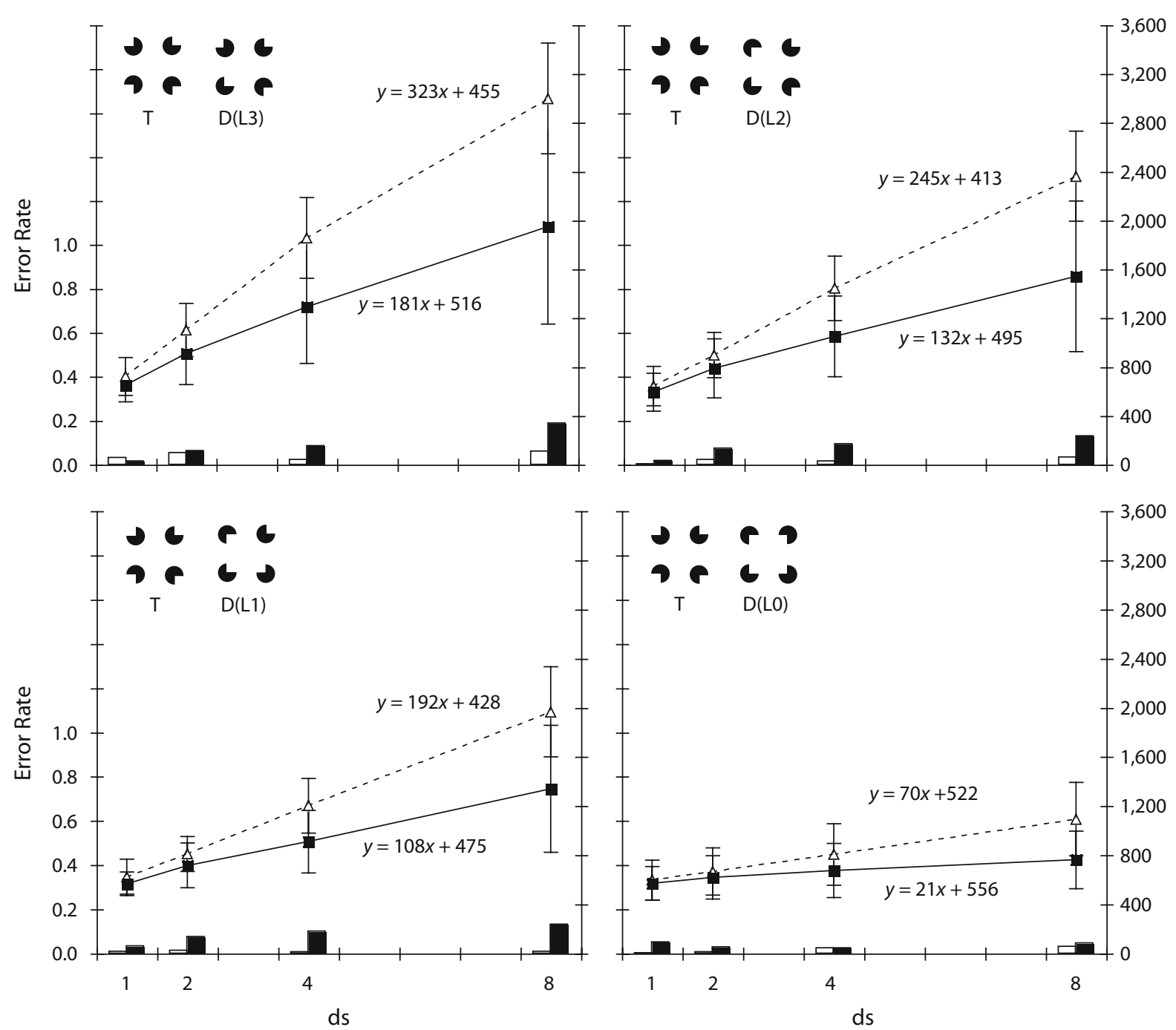

Figure 9. Mean RTs (with associated SDs) and error rates in Experiment 3 as a function of display size (ds), with three to zero local inducers that match the orientation with the target (top-left to bottom-right graphs, respectively). Each graph shows the prototype target (T) and an example of a distractor (D) and plots RTs and error rates separately for target-absent (dotted line, white bars) and target-present trials (solid line, black bars). In addition, the function for the best fitting straight line is given for each search RT function, with the search rate and base RT estimates.

local inducers. Thus, both RT and error-rate data showed similar trends, with no speed-accuracy tradeoffs evident.

Next, differences between searches at local and global object levels were examined by comparing Experiments 1 and 3 in a mixed ANOVA of the same design as that described above for the RT analysis. This ANOVA revealed a significant four-way interaction $[F(3,42)=3.42, p<.01]$, as was the case for the RT data. Errors - in particular, miss rates-were more frequent in Experiment 3 than in Experiment 1 , with interference resulting from distractor type variations leading to a larger increase in errors with display size in Experiment 3 as compared to Experiment 1. Thus, the pattern of error results reinforces the RT effects.

Discussion. In Experiment 3, the change of the target from a globally to a locally defined configuration produced an outcome qualitatively similar to those in the previous experiments: The search time per item increased as more distractors matched the target in terms of inducer element orientation. However, considering search efficiency, a clear quantitative difference is evident: Response latencies and error rates were extremely sensitive to changes in the local orientation of the inducers. Search was clearly more impaired in Experiment 3 than in the previous experiments: There was a large increase in the time taken to process a configuration as the distractor elements increasingly matched the target elements in their orientations. This suggests that local-element processing does not support search as efficiently as coding at the global or object level. A possible explanation for this is that, at a local object level, each single inducer has to be inspected, whereas at a global object level, the formation of the illusory figure supports more efficient processing in terms of the number (i.e., effectively by reducing the number) of search-critical objects. 
Interestingly, search conditions with similar distractors in the present Experiments 1 and 3 yielded clear performance differences. In correspondence with studies of search asymmetries (e.g., Treisman \& Gormican, 1988), performance was more efficient when the target contained additional information relative to the distractors (i.e., a global illusory figure), than when the target was defined by the absence of additional information (i.e., no global object). In other words, search was slower for local as compared to global targets, even though a global representation in distractors, such as for $\mathrm{D}(\mathrm{L} 2)$ distractors, could conceivably have helped observers reject this configuration as a possible target. For example, the mean increase in search time per item for $\mathrm{D}(\mathrm{L} 2)$ distractors was $188.5 \mathrm{msec}$ in Experiment 3. However, the identical global-form distractor, D(2), in Experiment 1 resulted only in a mean increase in search time of $59 \mathrm{msec} /$ item. This indicates that search processes cannot switch between global and local levels, but that detection is tied to the level at which the target is currently defined.

\section{EXPERIMENT 4}

Experiments 1-3 showed that search is influenced by global attributes of the target configuration. Depending on critical aspects that define the target, both global and local levels may lead to interference. In Experiments 1 and 2 , the representation of global surface information was found to be a major source of interference in search for an illusory square. By contrast, in Experiment 3, search was revealed to be dependent on the local orientation of inducers. In Experiment 4, the global surface effect reported for Experiment 1 was investigated in more detail.

Experiment 4 was designed to examine the interference produced by distracting global object information when the number of potential targets in a given display is gradually increased. As in the previous experiments, participants were asked to search for a target Kanizsa figure. However, contrary to the previous experiments, displays always consisted of eight candidate groupings. Distractors were either baseline nonsquare (placeholder) distractors or global-form and global-border configurations as in Experiment 1. The number of global distractors presented together with the placeholders in a given display was systematically varied. This permitted search performance to be examined with a gradual increase of interfering global representations. At the same time, this manipulation of display characteristics permitted ruling out that display density plays a crucial role for the interference effects observed in the previous experiments, since the number of configurations was held constant across conditions.

\footnotetext{
Method

Experiment 4 was identical to Experiment 1, except that observers were always presented with an eight-element display in which a target Kanizsa square had to be detected. Distractor items could either be baseline placeholder configurations with inducers rotated outward by $180^{\circ}$ to suppress illusory figure formation - see $\mathrm{D}(0)$ distractors in Figure 4 - or, alternatively, one of four possible configurations displaying global stimulus characteristics: global-border and global-form distractors labeled $\mathrm{D}(1)$ and $\mathrm{D}(2)$. Thus, in a given
}

display, there were 0 to 7 distractors comprising global stimulus characteristics (the interference set). Interference distractors were presented at random positions within an eight-element display (see Figure 4B) together with 8 [7] to 0 [1] placeholder configurations for target-absent [target-present] trials, respectively. Eight paid observers ( 5 of whom were male, mean age 25.5 years) with normal or corrected-to-normal vision performed the experiment.

The experiment consisted of four sessions with eight blocks of 80 trials each. Each session presented one type of the interference distractors, counterbalanced across observers. In summary, the independent variables were target (T: present, absent), interference set (IS: $0,1,2,3,4,5,6,7$ configurations), and interference distractor (ID: GF1, GF2, GB1, GB2) types coding the number of illusory contours in global-form and global-border configurations (see Figure 10) with 40 trials per experimental condition. All other details were identical to the procedure described for Experiment 1.

\section{Results and Discussion}

RT analysis. Trials on which a response error was made $(2.7 \%)$ were removed from the data set prior to RT analysis. Figure 10 presents the mean correct RTs and the percentage of errors as a function of the interference set, separately for the different global-form and global-border interference distractors. As can be seen, as for Experiment 1 , the global-form distractor exhibited an increase in search times per interference distractor. By contrast, no comparable effect was present for global-border conditions.

The RT data were examined by a repeated measures ANOVA with the factors target, interference set, and interference distractors. This ANOVA revealed all effects to be significant [T, $F(1,7)=21.37, p<.01$; IS, $F(7,49)=$ $57.85, p<.001 ;$ ID $F(3,21)=15.14, p<.001 ; \mathrm{T} \times$ IS, $F(7,364)=10.77, p<.001$; T $\times$ ID, $F(3,364)=$ $77.27, p<.001$; IS $\times \mathrm{ID}, F(21,364)=18.02, p<.001$; $\mathrm{T} \times \mathrm{IS} \times \mathrm{ID}, F(21,364)=4.41, p<.001]$. Response latencies, especially in the target-absent case, displayed a steeper increase with an increase in the number of illusory contours in the global-form as compared to the global-border condition. Each additional contour in the global-form condition slowed search by 28.0 [80.0] msec per interfering distractor. By contrast, no slowing was observed for increases in illusory contours in the globalborder condition $(0.0[-1.0] \mathrm{msec} /$ interfering distractor). Consequently, this pattern of results mirrors the outcome of Experiment 1 in showing that interference results from global form, but not from borders.

In addition to this search asymmetry, the base RTs also showed a clear effect when examined in terms of the number of illusory contours in distractors: For global-form distractors, the addition of an illusory contour increased base RTs by $164.5 \mathrm{msec}$. Similarly, global-border distractors exhibited a base RT increase of $135.5 \mathrm{msec} /$ contour (see Figure 10, top and bottom graphs). A repeated measures ANOVA performed on outlier-corrected base RTs confirmed that this increase for one versus two illusory contours in distractors was significant $[F(1,15)=5.68$, $p<.04]$. This indicates that the illusory contours are processed to some extent for both global-form and globalborder configurations; however, only surface specifications in global-form configurations lead to changes in search efficiency. 

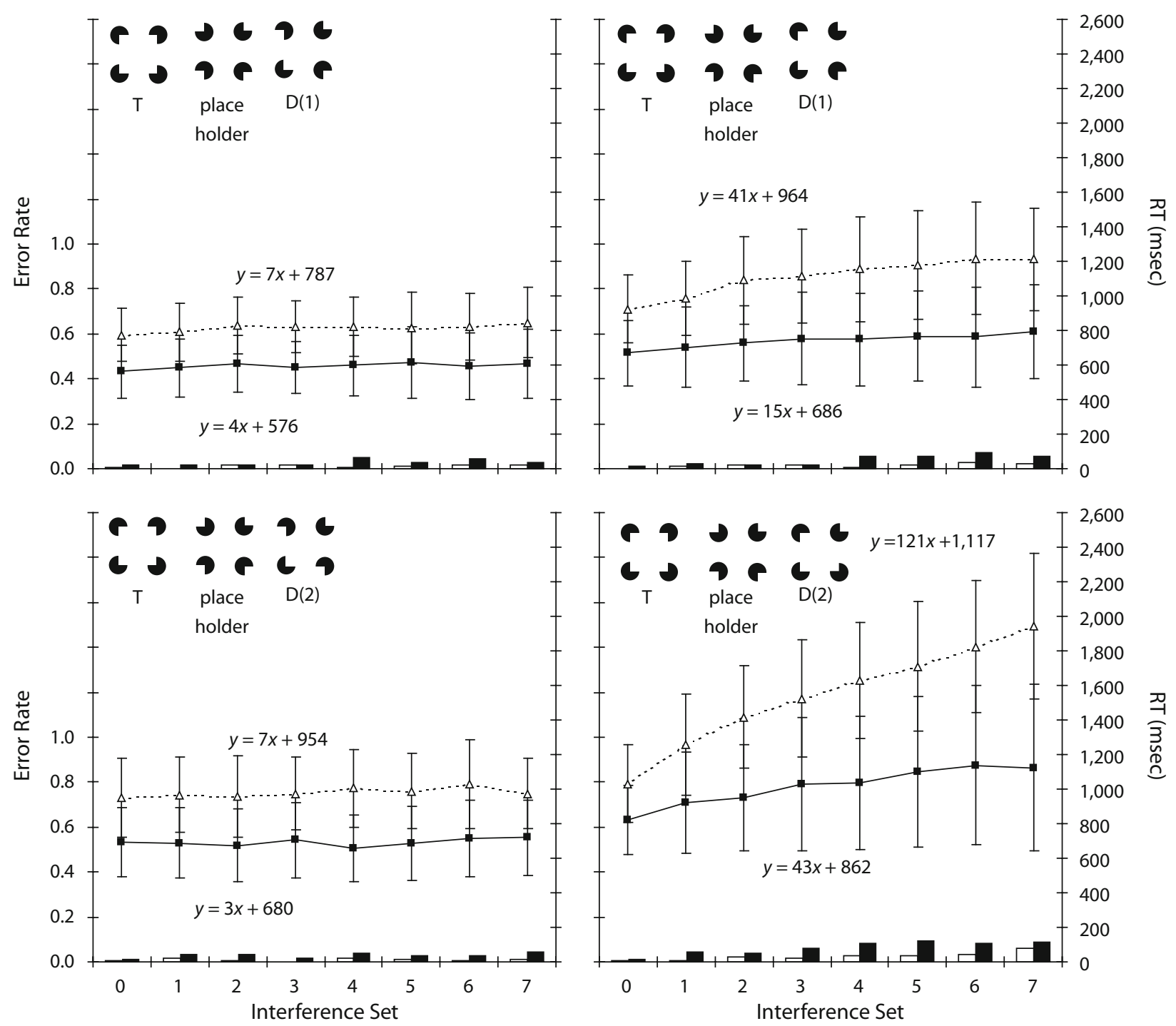

Figure 10. Mean RTs (with associated $S D$ s) and associated error rates as a function of the interference set in Experiment 4. Each graph plots a prototype target (T) and examples of placeholders and interference distractors (D) from the global-border (left column) and global-form condition (right column) with distractors comprising one versus two contours (top vs. bottom panels, respectively). RTs, SDs and error rates are plotted separately for target-absent (dotted line, white bars) and target-present trials (solid line, black bars). In addition, linear functions are given for each RT distribution depicting slopes and base RT estimates.

Error analysis. Response errors were relatively rare overall $(3.9 \%$ misses; $1.4 \%$ false alarms). The arcsinetransformed error data were analyzed by means of an identical ANOVA to that of the RT data. This analysis revealed all main effects and two-way interactions to be significant $[\mathrm{T}, F(1,7)=28.01, p<.01$; IS, $F(7,49)=8.61, p<.001$; ID, $F(3,21)=8.48, p<.001 ; \mathrm{T} \times \mathrm{IS}, F(7,364)=3.52$, $p<.01 ; \mathrm{T} \times \mathrm{ID}, F(3,364)=4.21, p<.01 ; \mathrm{IS} \times \mathrm{ID}$, $F(21,364)=1.89, p<.05]$. Errors - in particular, miss rates -increased with variations in the interference distractor and increases in the interference set. In the globalform condition, error rates were higher with an increase in the interference set than in the global-border condition. Speed-accuracy tradeoffs were not evident.

\section{Discussion}

Experiment 4 replicated the main outcome of Experiment 1 in showing that surface specifications exert a major influence on Kanizsa figure detection. Surfaces in global-form distractors interfered with search and led to an increase in search slopes. By contrast, no increase of search slopes was observable for global-border distractors, comparable to the outcome of the previous experiments. However, by extension to this replication of Experiment 1, variations in the base RTs indicate that contours are nonetheless processed. Each additional contour slowed mean base rate RTs by $150 \mathrm{msec}$, irrespective of global-form or global-border interference distractors. This indicates that both distractor types are processed in search; how- 
Table 1

Search Rate Increases (in Milliseconds) per Global Contour in Distractors for Experiments 1 and 2 and the Corresponding Increase in Search Rates per Local Inducer That Match in Orientation Between Target and Distractors

\begin{tabular}{|c|c|c|c|c|c|}
\hline & \multicolumn{2}{|c|}{ Experiment 1} & \multicolumn{2}{|c|}{ Experiment 2} & \multirow{2}{*}{$\begin{array}{c}\text { Experiment } \\
\text { Inducer }\end{array}$} \\
\hline & Border & Form & Form & Surface & \\
\hline Targ & 9.0 & 71.5 & 73.5 & 3.0 & 84.3 \\
\hline Target present & 3.0 & 34.0 & 32.5 & -2.0 & 53.3 \\
\hline Mean & 6.0 & 52.7 & 53.0 & 1.0 & 68.8 \\
\hline
\end{tabular}

Note-Increases were estimated as $[\mathrm{D}(n)-\mathrm{D}(0)] / n$, where $\mathrm{D}(n)$ is the search rate for a given distractor with $n=2$ global contours or $n=3$ local inducers.

ever, only the specification of surfaces yields an effect on search efficiency.

\section{GENERAL DISCUSSION}

The present set of experiments was conducted to reexamine results from previous studies investigating the role of completion processes in search for Kanizsa figures (Davis \& Driver, 1994; Grabowecky \& Treisman, 1989; Gurnsey et al., 1996). Our paradigm employed systematic variations of object attributes in distractors in order to identify critical figural properties for detection performance. In considering the illusory figure as a stimulus configuration comprising a hierarchy of global and local object attributes, these were varied to determine their relative impact on search performance.

Experiment 1 investigated the role of global border (i.e., contour) and form (i.e., contour plus surface) information for detection of an illusory square. The results suggested that interference between target and distractors originates from global surface specifications (in the global-form condition), whereas corresponding global contours alone did not give rise to comparable interference (see Table 1). As a follow-up, Experiment 2 was designed to isolate the critical aspects of global form attributes by manipulating contours independently of corresponding surface characteristics. The results indicated that global contours are computed independently of the specification of the global surface: Search efficiency varied only as a function of surface specification, and not with variations of the global contour (see Table 1). Thus, Experiments 1 and 2 demonstrated a specific role for the global surface in detecting a target Kanizsa figure. Following this, Experiment 3 examined the effects of local attributes on search efficiency. The results showed that, when observers were asked to detect a nonsquare target defined by the orientation of local inducers, search efficiency varied according to the extent to which the inducers in distractors matched the orientation of the target inducers (see Table 1). While the pattern of interference was qualitatively comparable with search for a globally defined target, search was much more inefficient, that is, search rates decreased more markedly with variations of local attributes as compared to variations in global object properties. Finally, Experiment 4 was designed to examine how a continuous increase in interfering global information influences search performance.
The results showed that search efficiency was determined by the global surface, as in Experiment 1. However, contour information was found to also influence base RTs, suggesting that global contour information is nevertheless encoded in search.

A consistent outcome of all experiments is the demonstration of a competitive target-distractor interaction in search performance. The results show that groupings comprising a hierarchical organization interact during search at that level which critically defines the target. Depending on the dominant level of organization of the target, interference can arise at either the global-surface or the local-inducer level of representation. For Experiments 1,2 , and 4, search for a target Kanizsa figure interfered mostly with global surface information in distractors. By contrast, in Experiment 3, a target defined by the local orientation of inducers interfered mostly with corresponding local matches of inducer orientation in distractors.

Importantly, a comparison of detection performance for a global versus a local target configuration reveals clear differences: Detection of a local configuration was by far more vulnerable to interfering distractors than detection of a global configuration. As can be seen from Figure 11, performance is markedly different between search for a global target (left panel, Experiment 1) and search for a local target (right panel, Experiment 3) among identical distractor configurations. Note that the number of symmetry axes and other indices of figural complexity (see below) are equal for the targets and distractors in both conditions. Nevertheless, search for a local target configuration is far less efficient than search for a global configuration [search rates of 188.5 vs. $59.0 \mathrm{msec} /$ item; $t(14)=$ $6.01, p<.001]$. This search asymmetry is hard to explain in terms of an account based on the computation of local element similarities, that is, in terms of interference being a monotonic function of the number of features shared between the target and the distractors (see, e.g., Duncan \& Humphreys, 1989). Instead, the marked search rate difference between the two conditions is likely to reflect a difference in processing across the hierarchy of processing levels involved. In this view, the Kanizsa target square is detected on the basis of global surface properties, whereas detection of the nonsquare configuration relies on orientation differences between local inducer elements. In other words, search for a local target configuration is slow because grouping operations do not apply as efficiently as in search for a global configuration.

An alternative account to interference arising at differential levels of hierarchical processing might be an explanation in terms of figural complexity. According to this view, search efficiency would vary as a function of figural complexities in target and distractor configurations (see Rauschenberger \& Yantis, 2006, for a similar proposal). To investigate this, for each configuration employed in the present experiments, figural complexity was estimated by counting the possible rotations and reflections of a given stimulus configuration (the so-called R\&R operations; see Garner \& Clement, 1963). However, a comparison of R\&R operations (Table 2) with variations in search slopes for Experiments 1-3 failed to show a satisfactory degree 

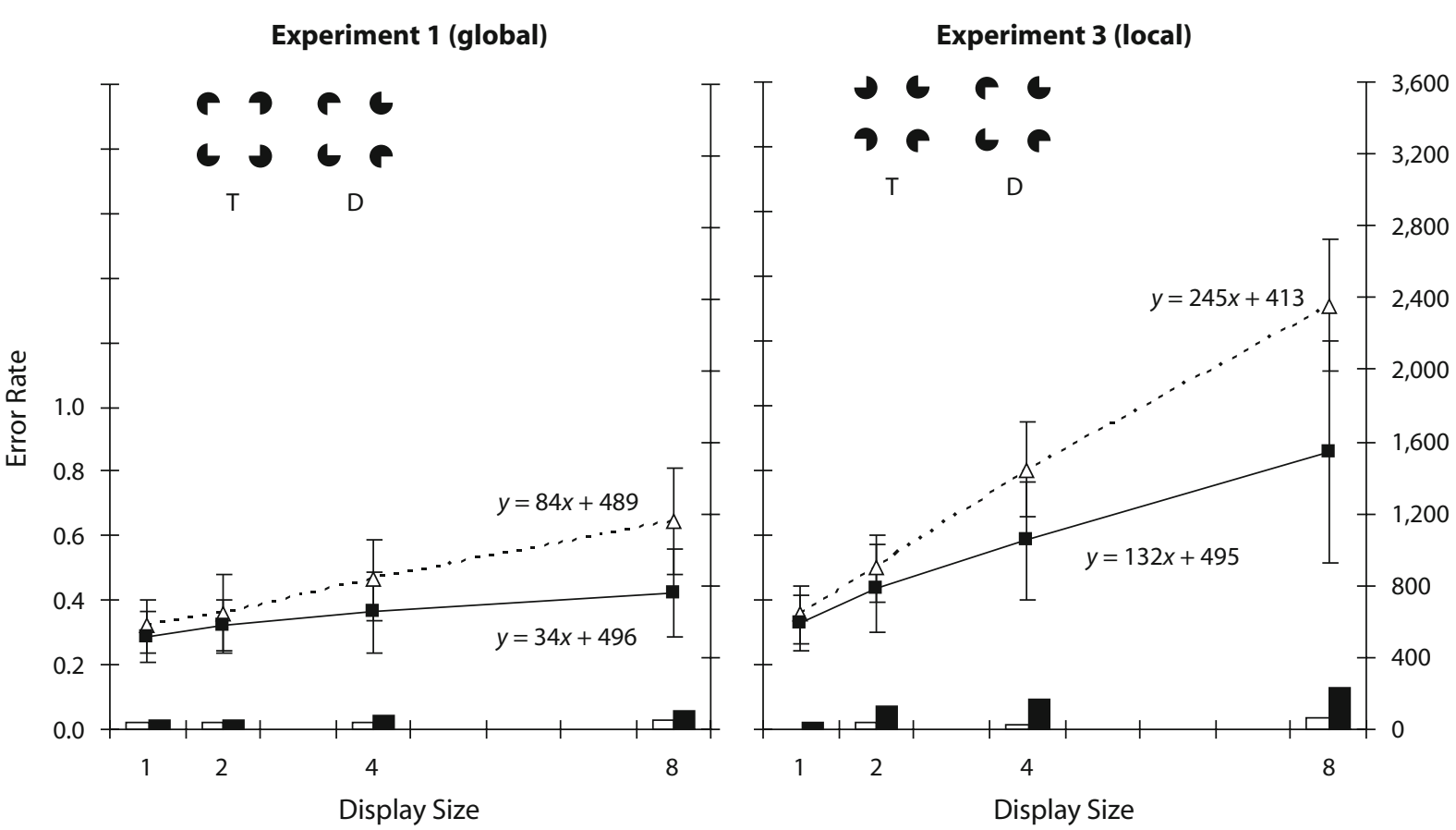

꼭

Figure 11. Comparison of search for global versus local target configurations. Search for a global target (Experiment 1) is by far more efficient than search for a local target (Experiment 3), even though distractor configurations are the same.

of covariation $(r=.20 ; p=.44)$. Instead, for Experiments 1 and 2 , the specification of surface portions in distractors was related to variations of search slopes $(r=.92, p<$ .001 , for a correlation between slopes and the number of global surface quadrants in distractors). For Experiment 3, a similar relationship was observable when comparing local inducer orientation matches with corresponding slopes $(r=.98, p<.01$, for a correlation between slopes and the number of local inducer matches in distractors). In summary, the amount of hierarchical object information in distractors covaries strongly with measures of search performance, whereas the figural complexity of distractors does not. This dissociation provides further support for the role of specific figural attributes in determining search efficiency in the present experiments.

A second alternative to explain the present set of results would be an account that relates search performance to configural size variations. In this view, the target Kanizsa

Table 2

Counts of Possible Reflections and Rotations per Global Contour in Target and Distractors for Experiments 1 and 2 and Corresponding Counts of R\&R Operations per Local Inducers That Match in Orientation Between Target and Distractors in Experiment 3

\begin{tabular}{lcccccccc}
\hline & \multicolumn{2}{c}{ Experiment 1 } & & \multicolumn{2}{c}{ Experiment 2 } & & \multicolumn{2}{c}{ Experiment 3 } \\
\cline { 2 - 3 } \cline { 7 - 8 } \cline { 7 - 8 } & Border & Form & & Form & Surface & & \multicolumn{2}{r}{ Inducer } \\
\hline Target & 1 & 1 & & 1 & 1 & & Target & 1 \\
D(0) & 1 & 1 & & 1 & 2 & & D(L0) & 1 \\
D(1) & 8 & 4 & & 4 & 8 & & D(L1) & 4 \\
D(2) & 4 & 4 & & 4 & 2 & & D(L2) & 4 \\
& & & & & & & D(L3) & 4 \\
\hline
\end{tabular}

Note-For a discussion of reflections and rotations (R\&R operations), see Garner and Clement (1963). figure would be detected efficiently because the open region in the center of the configuration is larger than that of the baseline distractor [D(1); see Figure 4]. Thus, search in the baseline condition would be efficient because the target exhibits a large open region, while the distractors display a comparably smaller central region. Accordingly, as the global surface in distractors increases $[\mathrm{D}(1)$ and $\mathrm{D}(2)$; see Figure 4A], the size of the central region of the distractor configurations becomes larger as well, increasing the similarity to the target. Importantly, this explanation does away with the need to consider the effects of global object properties. Instead, a simple size difference in the central open region between target and distractors would be sufficient to explain the pattern of results. Nevertheless, in a previous study, we have tested the influence of size variations empirically employing displays that presented collinear line junctions that varied in the extent to which junctions formed a square grouping (Conci et al., 2007; Experiment 4). Our data showed no indication of a size effect on search performance: Search efficiency was independent from variations of the size of the open region in distractors (see Figure 12). Consequently, this pattern of effects clearly shows that (minor) variations of configuration size are not sufficient to explain variations of search performance for the present results.

The present experiments revealed search for a Kanizsa figure to be dependent on processing at the global level of stimulus organization for efficiency, in particular, global surface information turned out to be the critical determinant of response latencies and error rates. This strong influence of global surface information contrasts with the absence of a display size-dependent influence of global contours. In this respect, detection of an illusory figure 


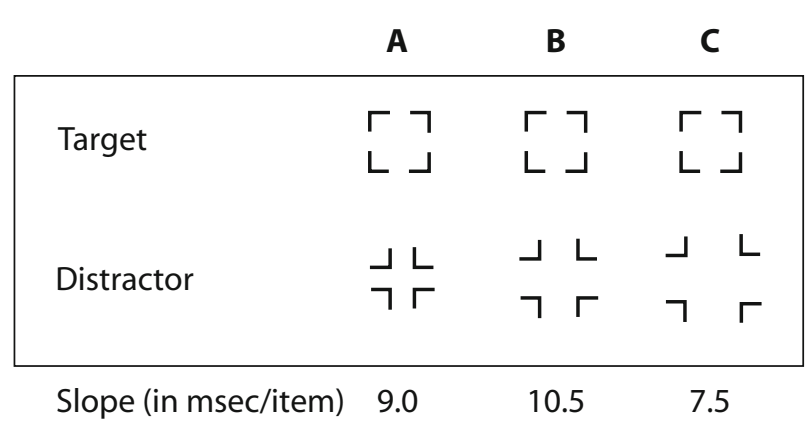

Figure 12. Effects of configural size variations in distractors on detection of a collinear target configuration. Targets could be (A) larger, (B) equally large, or (C) smaller than corresponding examples of baseline distractor configurations. Results indicated that the mean search rates on target-present trials (see bottom line) did not differ significantly from each other, indicating that search performance was independent of variations in the size of the enclosed regions in target and distractors. Adapted from Conci, Müller, and Elliott (2007, Experiment 4). Copyright 2007, Psychonomic Society, Inc.

shows a precedence of specific global object properties over local attributes consistent with a number of other types of stimulus configuration (He \& Nakayama, 1992; Navon, 1977; Rauschenberger \& Yantis, 2001; Rensink \& Enns, 1995; 1998). However, unlike Davis and Driver (1994) who had suggested an influence of the global contours, the present results argue that it is the surface, rather than the contour, that guides search. Thus, no direct link can be established between the outcome of visual search experiments and physiological studies that show specific responses to illusory contours in V2 (Lee \& Nguyen, 2001; Peterhans \& von der Heydt, 1991; von der Heydt et al., 1984) — a point that has been made previously by Gurnsey et al. (1996).

From the point of view of the computational model, it might be assumed that the processes of contour completion and surface filling-in are achieved by separate systems (Grossberg \& Mingolla, 1985, 1987). The emphasis on surface information in the present experiments supports this view and suggests that the filling-in of surfaces is achieved before a precise boundary has been computed (Stanley \& Rubin, 2003). This in turn indicates the importance of extracting salient regions in the rapid analysis of a visual scene. Indeed, psychophysical evidence suggests that global surface properties of objects can capture attentional resources (Rauschenberger \& Yantis, 2001), with search performance relying in many cases on a completed representation of object fragments (Davis \& Driver, 1998; He \& Nakayama, 1992; Rensink \& Enns, 1998).

In fact, in agreement with previous research on the role of region segmentation processes for the extraction of Kanizsa figures (e.g., Stanley \& Rubin, 2003), the present set of experiments suggests that surface properties are initially computed at a relatively coarse level to guide search. A comparison of configuration types that either did or did not elicit a global illusory figure shows that it is not necessary for surface-based representations to exhibit an integrated global object representation (see Figure 3B, pro- cessing step Number 4) in order to guide search. Rather, in the present experiments, search for an illusory Kanizsa square appears to follow similar principles as search for a collinear square configuration that does not elicit a global illusory figure (Conci et al., 2007). Consequently, surface representations do not need to be constructed in detail such that they comprise depth information or explicit contour representations. Instead, the computation of a crude salient region by means of global filling-in processes (see Figure 3B, processing step Number 3) appears to be sufficient to guide search, before an exact specification of an illusory figure becomes available.

From a physiological perspective, mechanisms capable of determining salient regions might be represented in a recurrent network which operates at multiple levels of processing across the visual scene (Lee \& Nguyen, 2001; Roelfsma, et al., 2002), integrating surface information and extracting their boundaries. In search, a recurrent network of this sort may compute various distinct attributes of an object (local inducers, global contours, global surfaces) at various levels of processing in the ventral stream. However, in order to discern the presence of an illusory figure in a complex search environment, it is sufficient to compute a single relevant attribute (i.e., its surface) to successfully detect the target.

\section{AUTHOR NOTE}

This work was supported by German Research Foundation (DFG) Project Grants MU 1564/2 and EL 248/2. We thank Doerthe Seifert, Pilar Gamez-Moreno, and Ayala Strulson for help with running the experiments, and Greg Davis, Jim Enns, and an anonymous reviewer for valuable comments on an earlier draft of this manuscript. Correspondence concerning this article should be addressed to M. Conci, Allgemeine und Experimentelle Psychologie, Department Psychologie, Ludwig-Maximilians-Universität, Leopoldstr. 13, D-80802 Munich, Germany (e-mail: conci@psy.uni-muenchen.de).

\section{REFERENCES}

Conci, M., Gramann, K., Müller, H. J., \& Elliott, M. A. (2006) Electrophysiological correlates of similarity-based interference during detection of visual forms. Journal of Cognitive Neuroscience, 18, 880-888.

Conci, M., Müller, H. J., \& Elliott, M. A. (2007). Closure of salient regions determines search for a collinear target configuration. Perception \& Psychophysics, 69, 32-47.

DAVIS, G., \& Driver, J. (1994). Parallel detection of Kanizsa subjective figures in the human visual system. Nature, 371, 291-293.

DAVIS, G., \& DrIVER, J. (1998). Kanizsa subjective figures can act as occluding surfaces at parallel stages of visual search. Journal of Experimental Psychology: Human Perception \& Performance, 24, 169-184.

Duncan, J., \& Humphreys, G. W. (1989). Visual search and stimulus similarity. Psychological Review, 96, 433-458.

EnNs, J. T., \& Kingstone, A. (1995). Access to global and local properties in visual search to compound stimuli. Psychological Science, 6, 283-291.

Ffytche, D. H., \& ZeKI, S. (1996). Brain activity related to the perception of illusory contours. NeuroImage, 3, 104-108.

Garner, W. R., \& Clement, D. E. (1963). Goodness of pattern and pattern uncertainty. Journal of Verbal Learning \& Verbal Behavior, 2, 446-452.

Grabowecky, M., \& Treisman, A. M. (1989). Attention and fixation in subjective contour perception. Investigative Ophthalmology \& Visual Science, 30, 457.

GrossberG, S. (2000). The complementary brain: Unifying brain dynamics and modularity. Trends in Cognitive Sciences, 4, 233-246. 
Grossberg, S., \& Mingolla, E. (1985). Neural dynamics of form perception: Boundary completion, illusory figures and neon color spreading. Psychological Review, 92, 173-211.

Grossberg, S., \& Mingolla, E. (1987). The role of illusory contours in visual segmentation. In S. Petry \& G. E. Meyer (Eds.), The perception of illusory contours (pp. 116-125). New York: Springer.

Gurnsey, R., Humphrey, G. K., \& Kapitan, P. (1992). Parallel discrimination of subjective contours defined by offset gratings. Perception \& Psychophysics, 52, 263-276.

Gurnsey, R., Poirier, F. J. A. M., \& Gascon, E. (1996). There is no evidence that Kanizsa-type subjective contours can be detected in parallel. Perception, 25, 861-874.

He, Z. J., \& NaKayama, K. (1992). Surfaces versus features in visual search. Nature, 359, 231-233.

Hirsch, J., DE la Paz, R. L., Relkin, N. R., Victor, J., Kim, K., Li, T., ET AL. (1995). Illusory contours activate specific regions in human visual cortex: Evidence from functional magnetic resonance imaging. Proceedings of the National Academy of Sciences, 92, 6469-6473.

Hubel, D. H., \& Wiesel, T. N. (1968). Receptive fields and functional architecture of monkey striate cortex. Journal of Physiology, 195 215-243.

KANIZSA, G. (1955). Margini quasi-percettivi in campi con stimolazione omogenea. [Quasi-perceptional margins in homogeneously stimulated fields.]. Rivista di Psycologia, 49, 7-30.

LeE, T. S., \& NGuYen, M. (2001). Dynamics of contour formation in the early visual cortex. Proceedings of the National Academy of Sciences, 98, 1907-1911.

LESHER, G. W. (1995). Illusory contours: Towards a neurally based perceptual theory. Psychonomic Bulletin \& Review, 2, 279-321.

Murray, M. M., Wylie, G. R., Higgins, B. A., Javitt, D.C., Schroeder, C. E., \& Foxe, J. J. (2002). The spatiotemporal dynamics of illusory contour processing: Combined high-density electrical mapping, source analysis, and functional magnetic resonance imaging. Journal of Neuroscience, 22, 5055-5073.
NAVON, D. (1977). Forest before trees: The precedence of global features in visual perception. Cognitive Psychology, 9, 353-383.

Palmer, S. E., Brooks, J. L., \& Nelson, R. (2003). When does grouping happen? Acta Psychologica, 114, 311-330.

Peterhans, E., \& von der Heydt, R. (1991). Subjective contours: Bridging the gap between psychophysics and physiology. Trends in Neurosciences, 14, 112-119.

RAuschenberger, R., \& YANTis, S. (2001). Attentional capture by globally defined objects. Perception \& Psychophysics, 63, 1250-1261.

Rauschenberger, R., \& Yantis, S. (2006). Perceptual encoding efficiency in visual search. Journal of Experimental Psychology: General, 135, 116-131.

Rensink, R. A., \& EnNs, J. T. (1995). Preemption effects in visual search: Evidence for low-level grouping. Psychological Review, 102, 101-130.

RENSINK, R. A., \& EnNs, J. T. (1998). Early completion of occluded objects. Vision Research, 28, 2489-2505.

Roelfsma, P. R., Lamme, V. A. F, Spekreijse, H., \& Bosch, H. (2002). Figure-ground segregation in a recurrent network architecture. Journal of Cognitive Neuroscience, 14, 525-537.

Spillmann, L., \& Dresp, B. (1995). Phenomena of illusory form: Can we bridge the gap between levels of explanation? Perception, 24, 1333-1364.

Stanley, D. A., \& Rubin, N. (2003). fMRI activation in response to illusory contours and salient regions in the human lateral occipital complex. Neuron, 37, 323-331.

Treisman, A. M., \& Gormican, S. (1988). Feature analysis in early vision: Evidence from search asymmetries. Psychological Review, 95, 15-48.

von der Heydt, R., Peterhans, E., \& Baumgartner, G. (1984). Illusory contours and cortical neuron responses. Science, 224, 1260-1262.

(Manuscript received October 31, 2006; revision accepted for publication May 3, 2007. ) 